\title{
EFFECT OF HCG WITH INTRAVAGINAL SPONGES ON REPRODUCTIVE PERFORMANCE IN AWASSI EWES DURING ESTRUS SYNCHRONIZATION
}

\author{
AMMAR ALLUGAMI*; MOHAMAD ZUHER AL-AHMAD ${ }^{* *}$ and NASOUH KHAZNADAR ${ }^{* * *}$ \\ * Master in artificel insemination and embryo transfer, Al-Baath University, Faculty of veterinary medicine, Hama, Syria. \\ ${ }^{* *}$ Reproductions of Artificiel Insemination \\ Artificiel insemination
}

Email: ammar.vetmed@hotmail.com

\section{ABSTRACT}

Received at: 31/3 /2014

Accepted: 17/5/2014
One hundred and nineteen Awassi ewes, 3 to 5 years old, treated with vaginal sponges. eCG and $\mathrm{hCG}$, were used to determine their response for estrus synchronization and pregnancy rates during anestrous season. Ewes were divided randomly into four groups. Vaginal sponges containing $20 \mathrm{mg}$ of FGA were inserted into all groups for 12 days, except the control group which stayed without any hormonal treatment. At the day of sponge withdrawal, ewes are divided into three groups: the first group (G1/29 ewes) were injected intramuscularly (im) with $2 \mathrm{ml}$ of saline. Ewes of the second group (G2/30 ewes) were injected (im) only with 500 IU of eCG. Ewes of the third group (G3/30 ewes) were injected (im) with 500 IU of eCG and $200 \mathrm{IU}$ of hCG. Results showed that there were significant differences $(\mathrm{P}<0.05)$ in estrus synchronization between ewes in G1 $(34.5 \%)$ comparatively with ewes of G2 and G3 (63.3\% and $86.7 \%$ respectively). General means of pregnancy rate were significantly higher $(\mathrm{P}<0.05)$ in $\mathrm{G} 2$ and $\mathrm{G} 3$ in regard to total number of all ewes in each group. The best lambing and twin average were recorded in G3 $(\mathrm{P}<0.05)$ which treatmed with $\mathrm{hCG}$. It was concluded that, using intravaginal sponges with the injection of $500 \mathrm{IU}$ of eCG in addition to the injection of $200 \mathrm{IU}$ of hCG at the day of withdrawal sponges, could be used for better estrus synchronization and increasing the reproductively in Awassi sheep under small holder rearing conditions, in nonbreeding season.

Keywords: HCG, Intravaginal sponges, estrus, Ewes.

\section{تأثير الـ hCG مع الإسفنجات المهبلية على الأداء التناسلي لأغنام العواسر السورية من خلال توقيت الشبقو}

\author{
عمار اللجمي ، محمد زهير الأحمد ، نصوح خزندار
}

Email: ammar.vetmed@hotmail.com

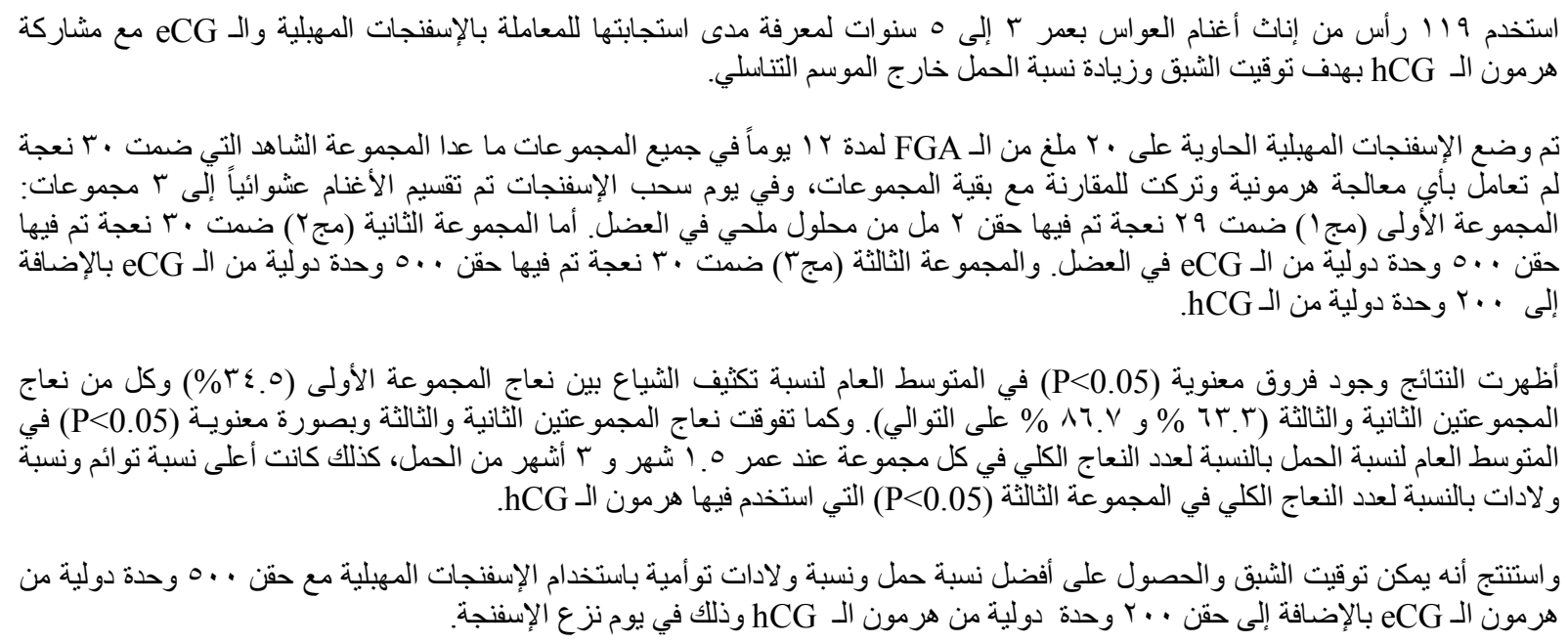




\section{INTRODUCTION \\ المقدمـة}

تعتبر الثروة الحيو انية في القطر العربي السوري أحد الأسس التي يعتمد عليها الدخل القومي والثروة الغنمية إحدى الأعمدة الأساسية لها ويشها

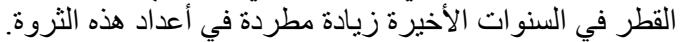

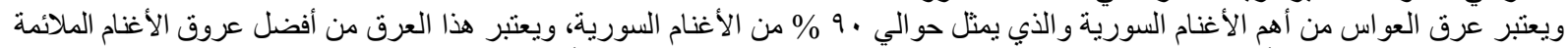

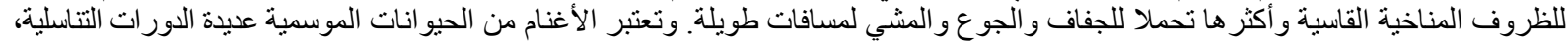

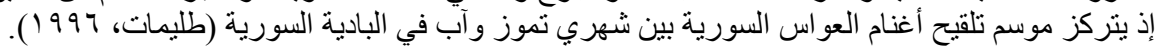

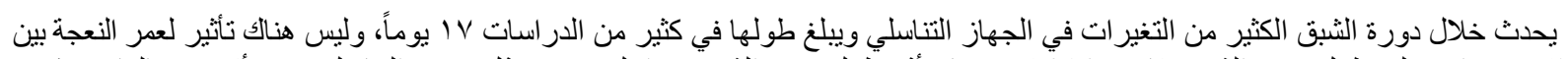

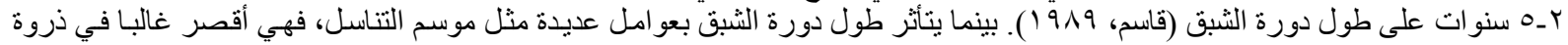

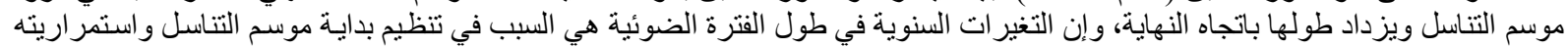

عند النعاج (Hafez, 1974).

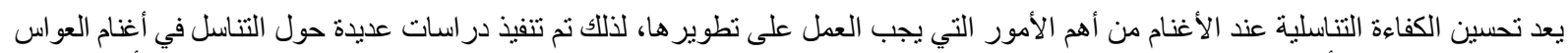

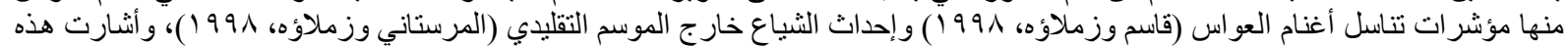

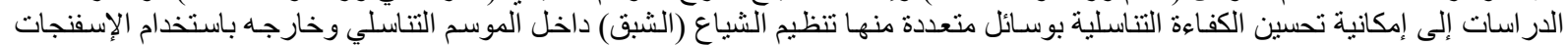

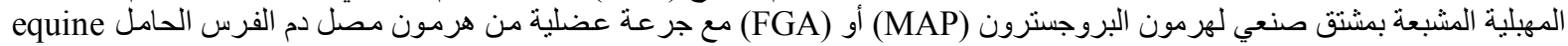
.(eCG) Chorionic Gonadotropin

عادةً يتم وضع الإسفنجات المهبلية لمدة 9 ـو 190 يوماً بالإضافة إلى استخدام الـ eCG خاصةً خارج الموسم التناسلي حيث يتم حقنه في يوم سحب الإسفنجات (Wildeus, 2000).

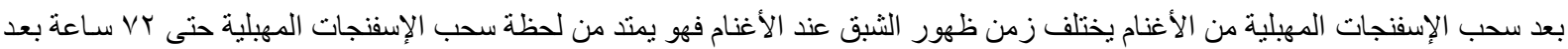

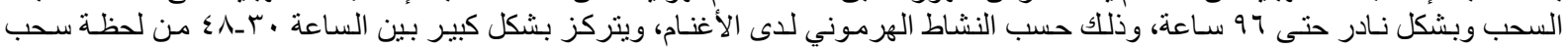

الإسفنجات (Ataman et al., 2006).

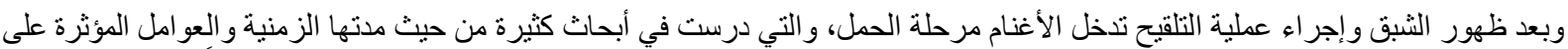

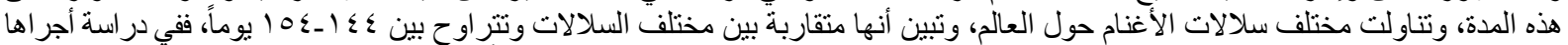
(Kassem et al., 1989)

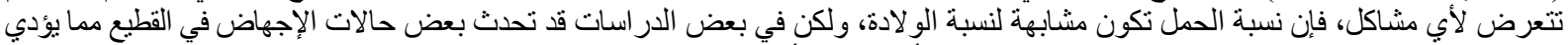

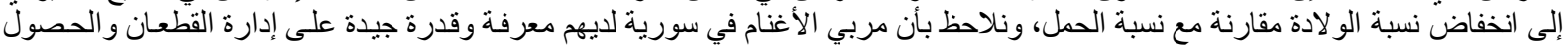

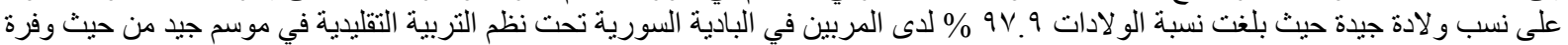

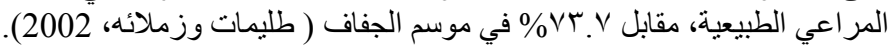

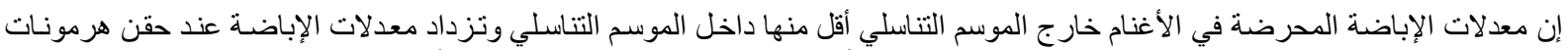

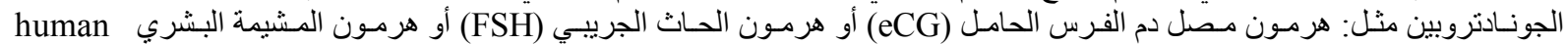
(hCG) Chorionic Gonadotropin

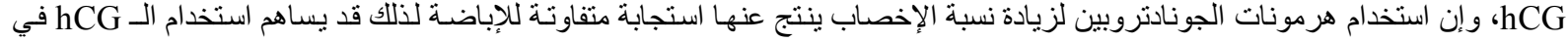

تحريض وتوقيت الإباضة (Knights et al., 2003).

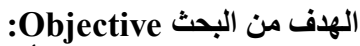

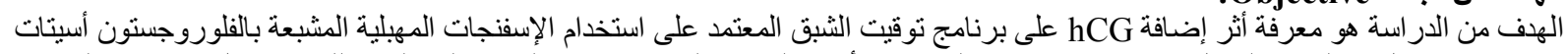

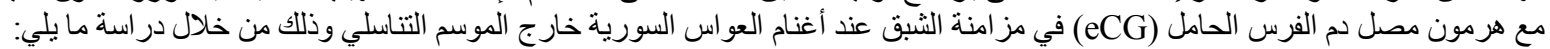

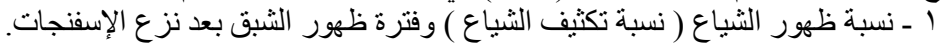

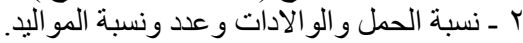

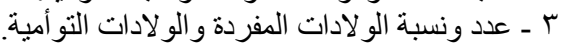

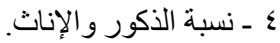

\section{MATERIALS and METHODS مواد وطر ائق العمل}

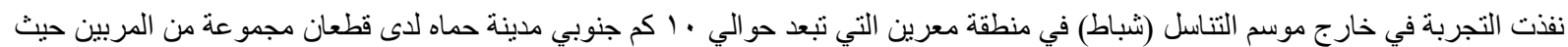

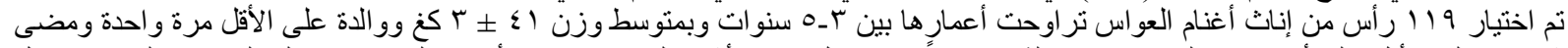

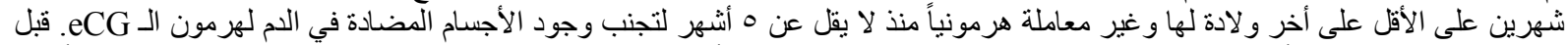

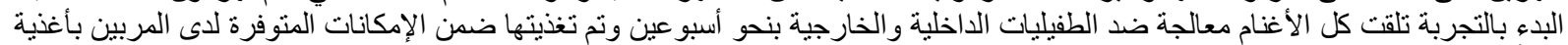

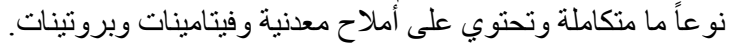




\section{Assiut Vet. Med. J. Vol. 60 No. 141 April 2014}

قسمـت الأغنـام بثكل عشو ائي إلى ؛ مجموعـات (الجدول رقم () بحيث تتضمن كل مجموعة كافة الفئات العمرية: تم توقيت الثبق في كافة

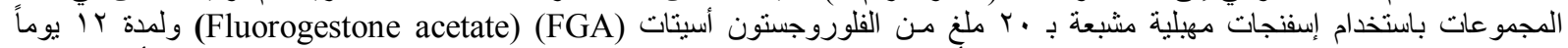

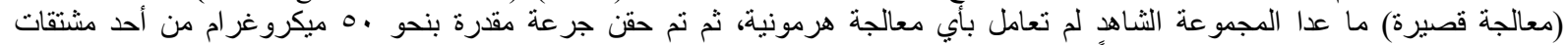

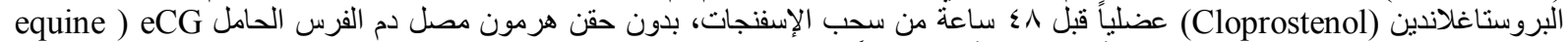
(Chorionic Gonadotropin

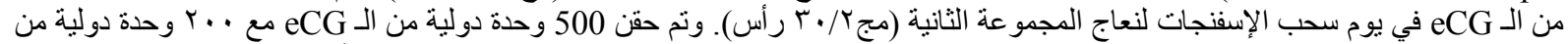

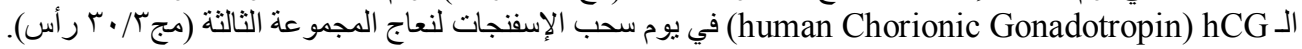

الجدول رقم 1: يوضح نو عية المعاملة الهرمونية في المجمو عات الأربعة.

\begin{tabular}{|c|c|c|c|c|}
\hline مجr & مجن & مجا & مج · (الثاهل) & نوعية المعالجة \\
\hline$r$. & $r$. & rq & $r \cdot$ & عدد نعاج المجموعة \\
\hline كا بوماً & كا بوماً & rا بوماً & - & (إسفنجات · r ملغ FGA) \\
\hline • 0 ميكروغرام & • 0 ميكروغر ام & • 0 ميكروغر ام. & - & بروستاغلاندين (كلوبروستينول) \\
\hline $500 \mathrm{UI}$ & $500 \mathrm{UI}$ & $0 \mathrm{UI}$ & - & eCG \\
\hline 200UI & 0 UI & $0 \mathrm{UI}$ & - & hCG \\
\hline
\end{tabular}

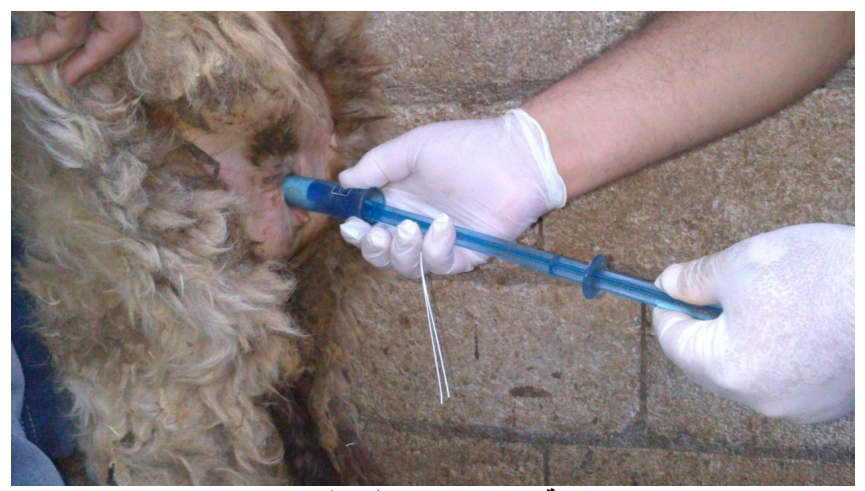

صورة رقم (1) توضح طريقة وضع الإسفنجات

تم بعد ذلك تسجيل فترة ظهور الثبق بعد نزع الإسفنجات عند نعاج المجمو عات الثلاث، ثم حسبت نسبة تكثيف الثياع من خلال تسجيل عدد الأغنام التي ظهر عليها الثبق صباحاً ومساءاً بفاصل 1 ساعات. وقد تم تطبيق المعادلة التالية لحساب نسبة تكثيف الثياع:

نسبة تكثيف الثنباع = عدد النعاج التي ظهر عليها الثنياع / مجموع عدد النعاج في التجربة × ...

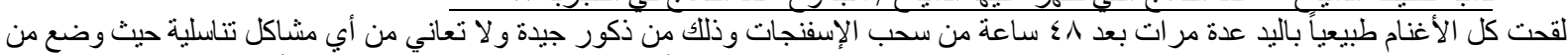

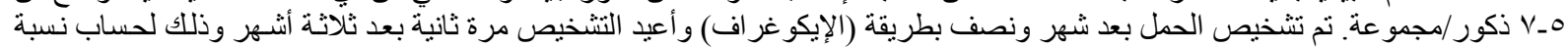

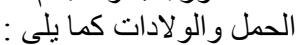

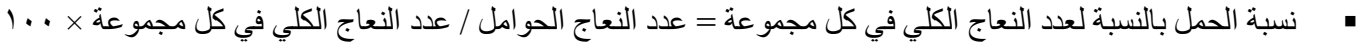

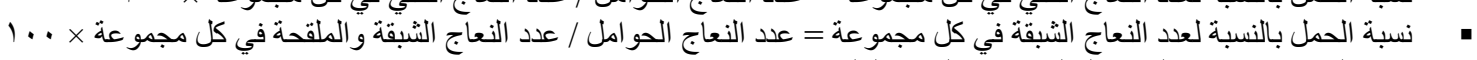

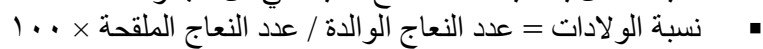

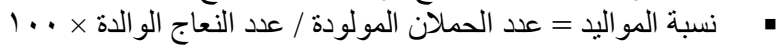

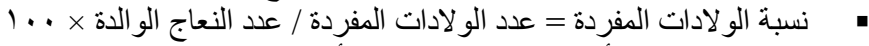

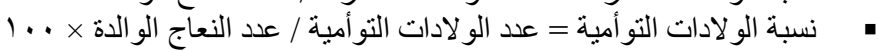

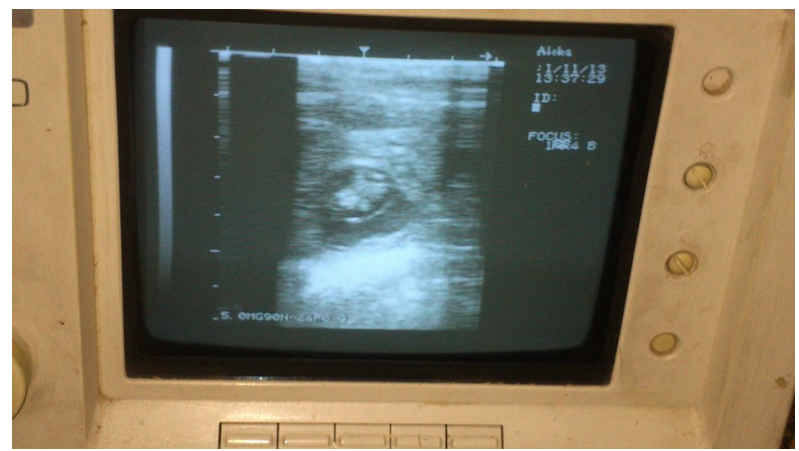

صورة رقم (r) نوضح تثخيص الحمل بجهاز الإيكو بعمر ه. اشهر من الحمل 
: Statistical analysis التحليل الإحصائي تم إجراء الوصف الإحصائي للنتائج باستخدام اختبار بيرسون مربع كاي Pearson's Chi Square لمقارنة مجاميع الدراسة بمعايير ها المختلفة،

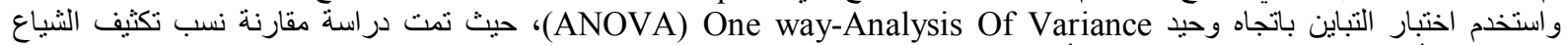

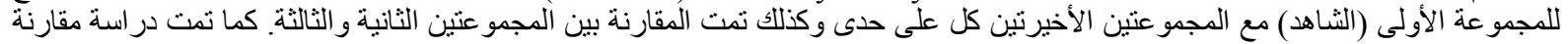

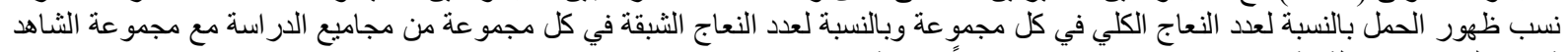

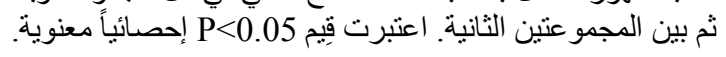

\section{RESULTS}

النتائسج

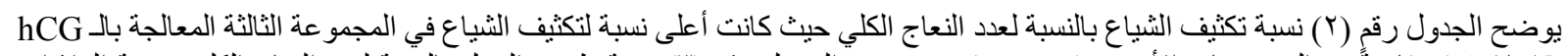

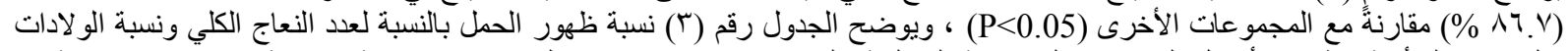

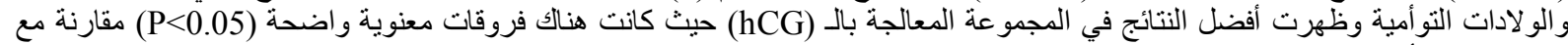
المجمو عات الأخرى.

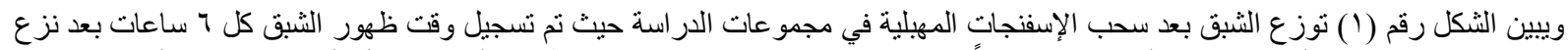

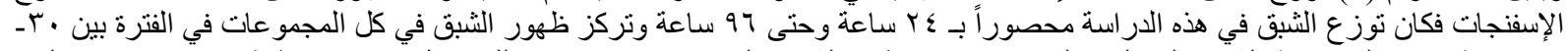

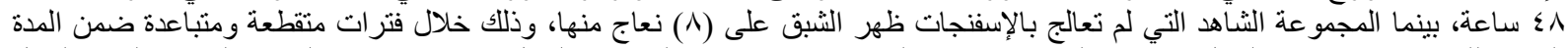

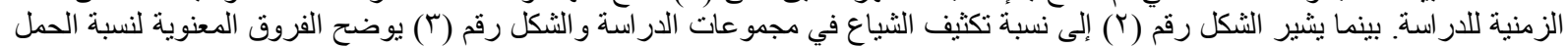
بين مجموعات الدراسة عند تثخيص الحمل بعمر م. الشهر بجهاز الإيكو ويظهر أنا أعلى نسبة للحمل (P<0.05) كانت في المجموعة الثالثة

الجدول رقم ץ بيين نسبة تكثيف الثياع في مجموعات الأغنام الددروسة.

\begin{tabular}{|c|c|c|c|}
\hline \multicolumn{2}{|c|}{ حدوث الشبق } & \multirow[t]{2}{*}{ عدد النعاج الكلي } & \multirow[t]{2}{*}{ وع المعاملة } \\
\hline نسبة تكثيف الثياع (\%) & عدد النعاج الثبقة والملقحة/الكلية & & \\
\hline$r 4.7$ & $r \cdot / \Lambda$ & $r \cdot$ & الشاهد \\
\hline aऍ & $r q / 1$. & rq & مج \\
\hline bat.r & $r \cdot / 19$ & $r \cdot$ & مجr \\
\hline 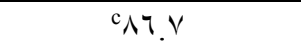 & $r \cdot / \Gamma T$ & $r \cdot$ & مجr \\
\hline
\end{tabular}

تشير الأحرف المختلفة في العمود الو احد إلى وجود فروقات معنوية بين المعاملات (P> • . • )

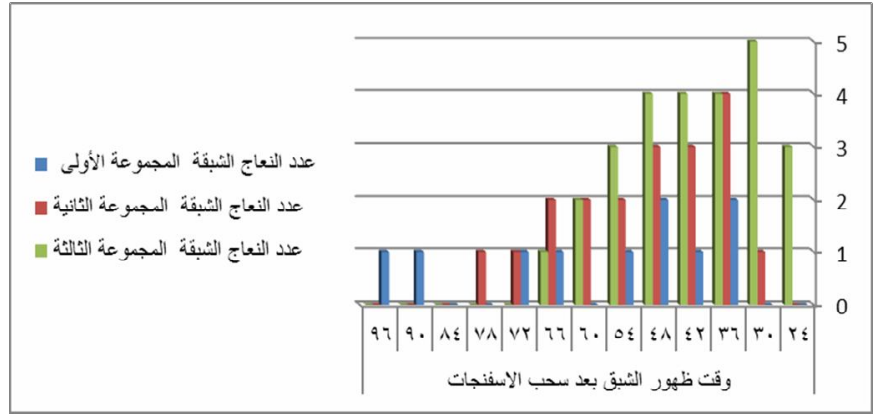

الشكل رقم 1: يبين توزع الثبق بعد سحب الإنفنجات المهبلية في مجموعات الدراسة

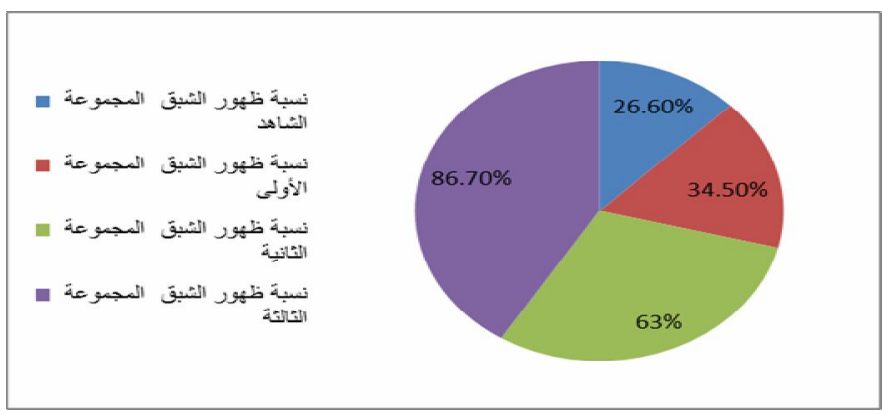

الثكل رقم r: بيين نسبة تكثيف الثباع في مجموعات الدراسة 


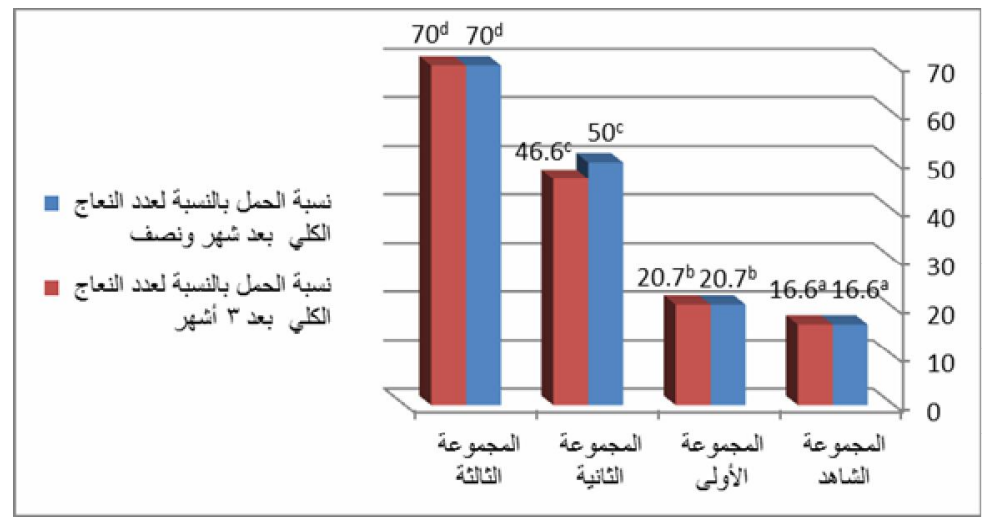

تشير الأحرف المختلفة في العمود الو احد إلى وجود فروقات معنوية بين المعاملات (P> • . • ). الشكل رقم بّ: نسبة ظهور الحمل بعمر شهر ونصف وعمر ثلاثة أشهر بالنسبة لعدد النعاج الكلي

جدول رقم بّ: يبين الأداء التناسلي لنعاج التجربة خارج الموسم التناسلي.

\begin{tabular}{|c|c|c|c|c|}
\hline FGA+eCG+hCG & FGA + eCG & FGA+Saline & Control & المعايير المدروسة \\
\hline$r \cdot$ & $r \cdot$ & rq & $r$. & عدد نعاج المجموعة \\
\hline${ }^{c}(\Lambda T . \vee) Y \tau$ & ${ }^{b}(7 \pi) 19$ & $\left.{ }^{a}(r \leq .0)\right)$. & 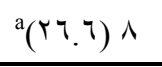 & 'ظهور الثبق (\%)' \\
\hline${ }^{b}(q . \vee) \curlyvee \wedge . \wedge$ & $(1 r . V) \leqslant 0 . r$ & $a(1 r .0) 0 \varepsilon$ & - & وقت ظهور الشبق بعد نزع الإسفنجات \\
\hline$\left.d^{d}(v \cdot) r\right)$ & ${ }^{c}(0 .)^{10}$ & ${ }^{b}\left(Y \cdot V^{V}\right)^{T}$ & ${ }^{a}(17.7)^{0}$ & الحمل بعر ๑. 1 شهر (\%) \\
\hline $\mathrm{d}(\mathrm{V} \cdot)^{\mathrm{r}} \mathrm{r}$ & $\left.c\left(\sum 7.7\right)\right) \leqslant$ & ${ }^{b}\left(Y \cdot . V^{r}{ }^{r}\right.$ & $\mathrm{a}(17.7)^{\circ}$ & الحمل بعر r شهر (\%) \\
\hline${ }^{\mathrm{bc}}(\lambda \cdot, \vee){ }^{\prime}$ & $\left.{ }^{b}(Y V, V)\right) \leq$ & $\mathrm{a}(\mathrm{l} \cdot)^{4}$ & $(4 r .0) 0$ & الولادات (\%) \\
\hline$b(19) \leqslant$ & ${ }^{a}(v .1)^{\prime}$ & $(\cdot) \cdot$ & $(\cdot) \cdot$ & الولادات التوأمية (\%) \\
\hline $\mathrm{b}(\varepsilon \cdot)^{1}$ & $(0, . r)^{\wedge}$ & $\mathrm{a}(77.7) \varepsilon$ & ${ }^{a}(\top \cdot)^{r}$ & نسبة المواليد الذكور (\%) \\
\hline${ }^{b c}(7 \cdot) 10$ & $\mathrm{~b}(\Sigma 7.7) \vee$ & a(rr.r) r & $(\varepsilon \cdot)^{r}$ & نسبة المو اليد الإناث (\%) \\
\hline
\end{tabular}

تشير الأحرف المختلفة في العمود الو احد إلى وجود فروقات معنوية بين المعاملات(P>0.05).

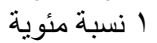
r المتوسط و الانحر اف المعياري

\section{DISCUSSION \\ المناقثة}

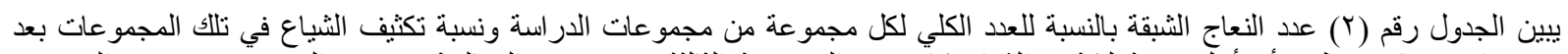

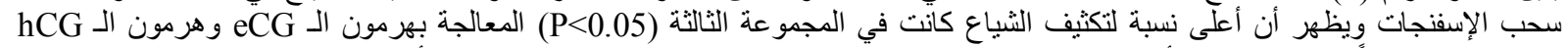

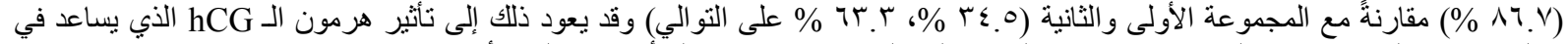

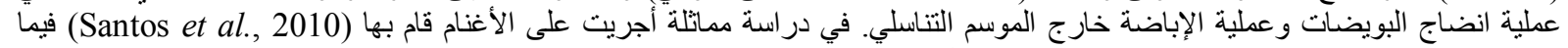

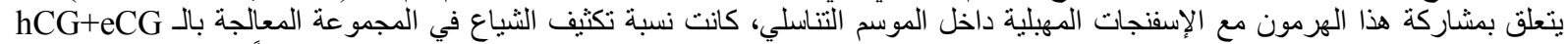

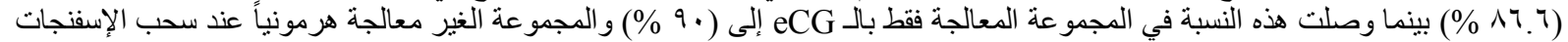
إلى (\% (\% \%

ولقد أشار (Farin et al., 1988) أن الـ hCG يحث على نمو وتطور جريبات المبيض ويزيد من افراز الأستروجين من الجريبات. وكانت نتائج

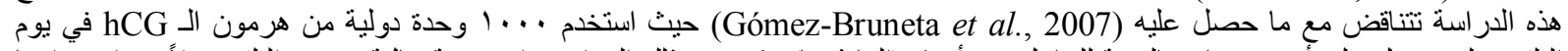

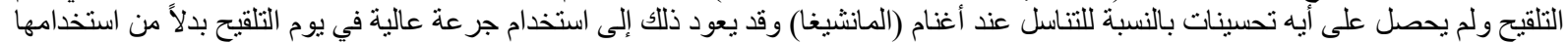

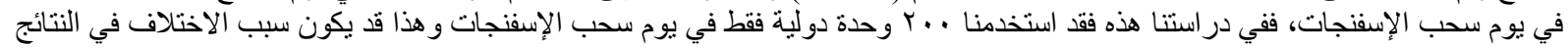




\section{Assiut Vet. Med. J. Vol. 60 No. 141 April 2014}

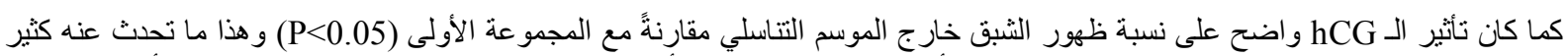

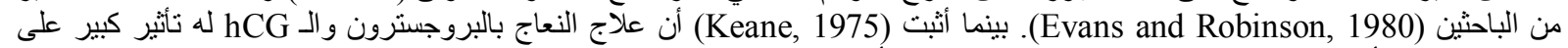

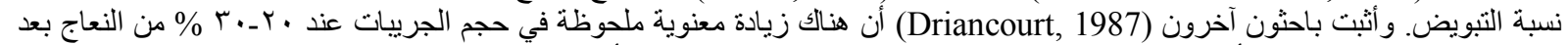

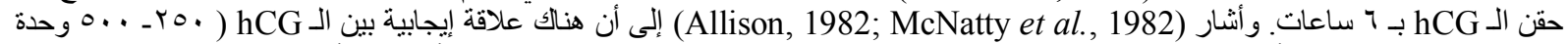

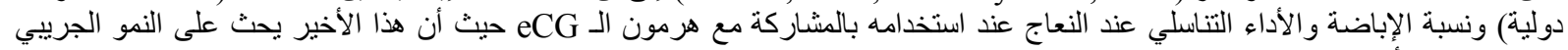
وزيادة افراز الأستروجين.

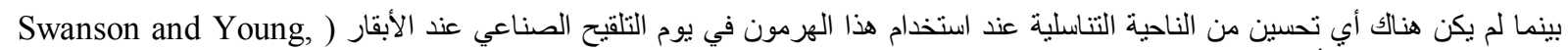

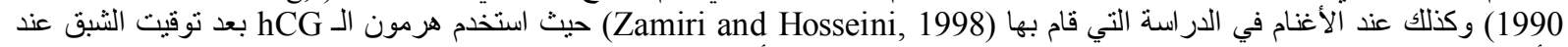

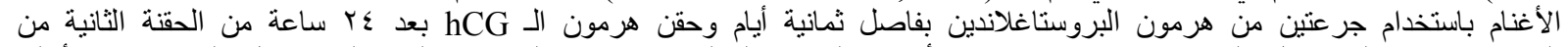

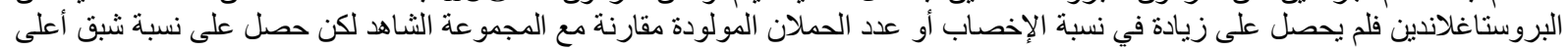
في المجموعة التي حقنت بـ . .0 وحدة دولية من الـ hCG.

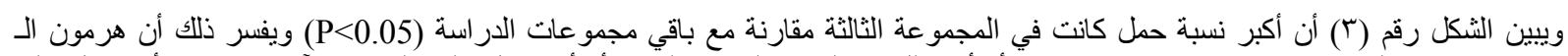

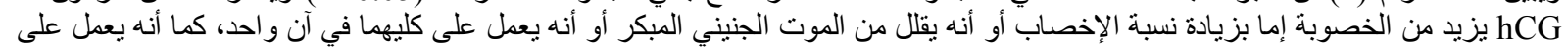

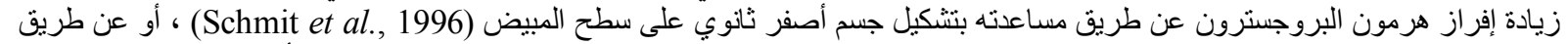

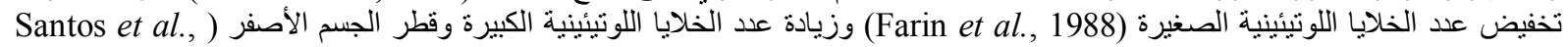

.(2001

إن المعدلات المنخفضة من البروجسترون في بداية ومنتصف الطور اللوتيئيني تعمل على إنخفاض نسبة الإخصاب حيث تسبب النمو غير الطبيعي

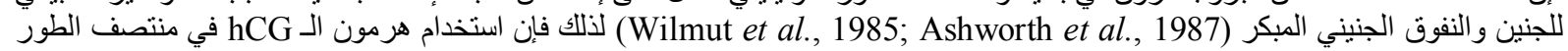

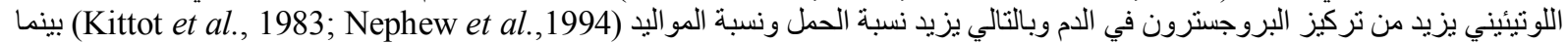

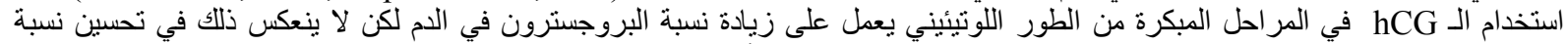

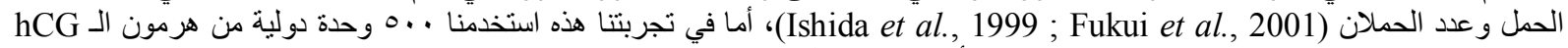
يوم سحب الإسفنجات في المجموعة الثالثة وحصلنا على أعلى نسبة حمل ونسبة ولادات.

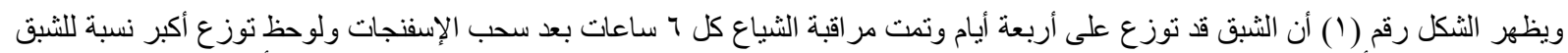

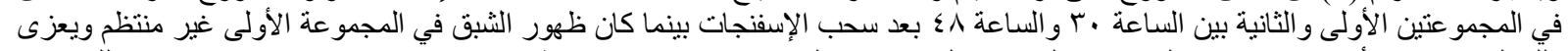

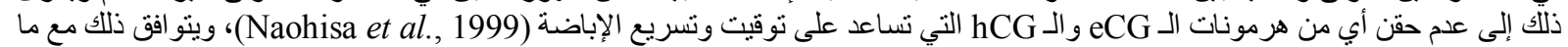

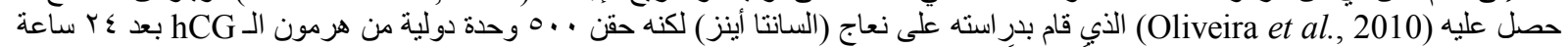

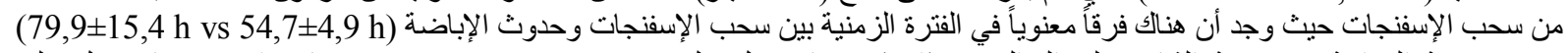

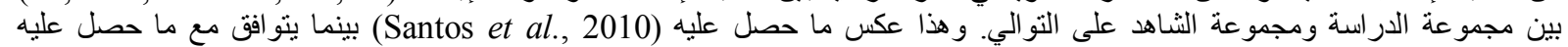

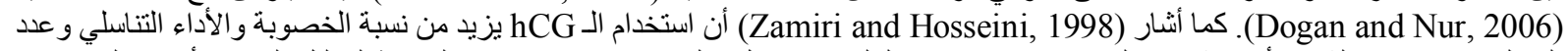
الدواليد عند توقيت الثبق بأحد مشتقات البروستاغلاندين وهو الكلوبروستينول والذي استخدمناه في هذه الدراسة لتحليل الجسم الأصفر الذي يمكن أن يكون فعالاً في لحظة سحب الإسفنجات (Gonzalez-Bulnes et al., 2002).

ويبين الجدول رقم (؟َ) نسب تثخيص الحمل بجهاز التصوير بالأمواج فوق الصوتية (الإيكوغراف) بعمر شهر ونصف ثم بعمر ب أشهر بالنسبة

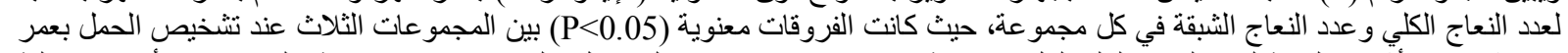

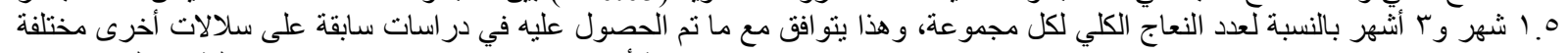

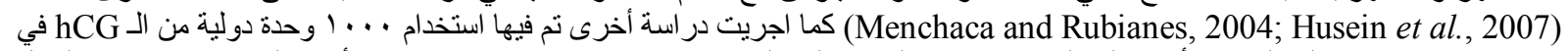

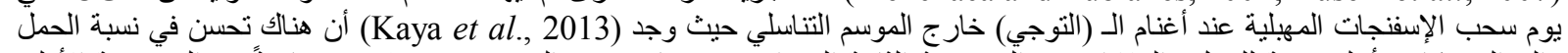

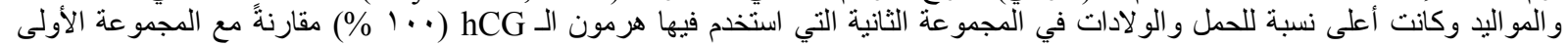

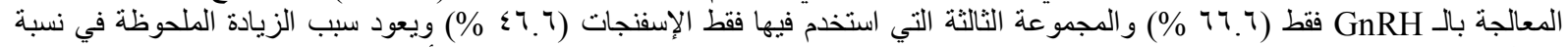

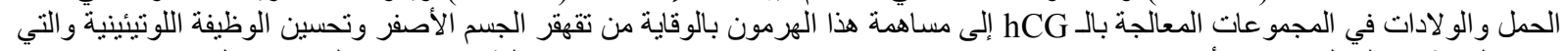

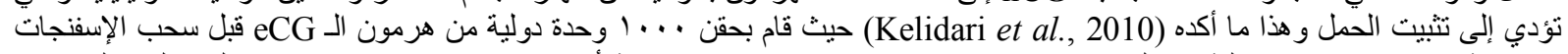

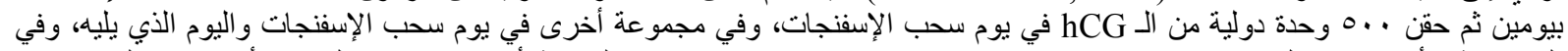

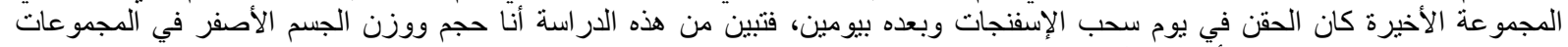

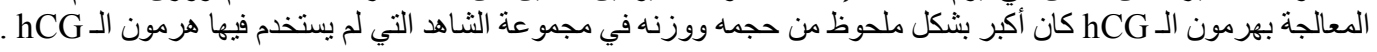

\section{CONCLUSIONS and RECOMMENDATION الاستنتاجات والتوصيات}

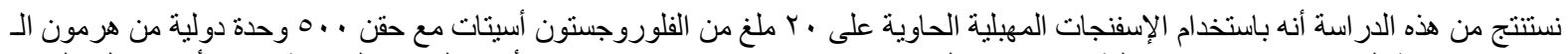

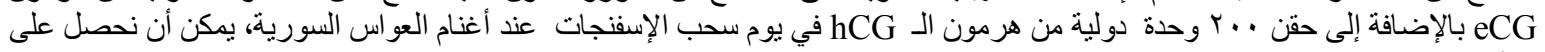
مايلي:

$$
\begin{aligned}
& \text { • توقيت جيد للشبق خارج الموسم التناسلي حيث يبدأ ظهور الثبق بوقت أقصر وخلال فترة زمنية أقل. } \\
& \text { • الحصول على نسبة عالية لتكثيف الثياع. } \\
& \text { • • الحصول على نسبة حمل أفضل. }
\end{aligned}
$$

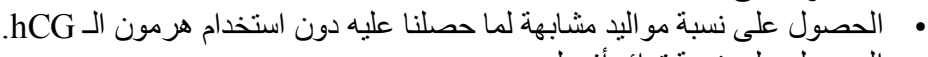

$$
\begin{aligned}
& \text { • الحصول على نسبة تو ائم أفضل. }
\end{aligned}
$$




\section{REFERENCES}

المراجـع

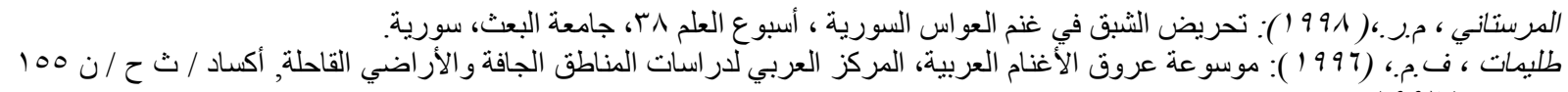

$$
\begin{aligned}
& .1997 /
\end{aligned}
$$

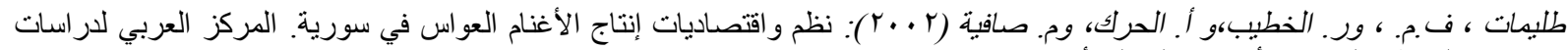

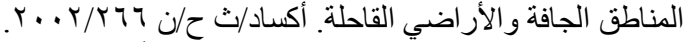

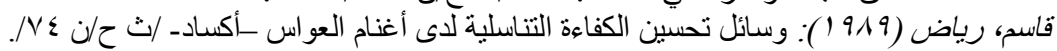

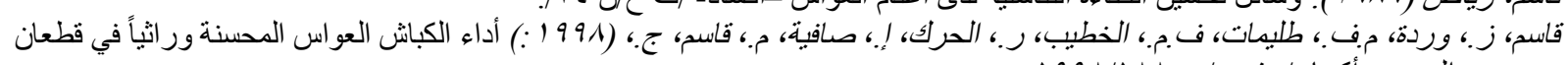

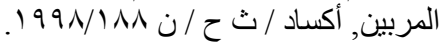

Allison, A.J. (1982): Technique of modifying reproductive performance in sheep production: breeding and production. (ed.) Wicham, G.A. and Mc Donald, M.F., New Zealand Institute of Agricultural Science, pp: 239-236.

Ashworth, C.J.; Wiltmut, I.; Springbett, A.J. and Webb, R. (1987): Effect of an inhibitor of $3 \beta$ hydroxysteroid dehydrogenase on progesterone secretion and embryo survival in sheep, J. Endocr., 112, $2 \overline{0} 5-213$.

Ataman, M.B.; Akoz, M. and Akman, O. (2006): Induction of syn-chronized oestrus in Akkaraman cross-bred ewes during breeding and anestrus seasons: the use of short-term and long-term progesterone treatments, Rev. Med. Vet., 50, 257-260.

Dogan, I. and Nur, Z. (2006): Different estrous induction methods during the non-breeding season in Kivircik ewes, Veterinarni Medicina, 51(4), 133-138.

Driancourt, M.A. (1987): Ovarian features contributing to the variability of PMSG-induced ovulation rate in sheep. J. Reprod. Fert., 80, 207-212.

Evans, G. and Robinson, T.J. (1980): The control of fertility in sheep. Endocrine and ovarian responses to progestagen PMSG treatment in breeding season and in anoestrus. J. Agric. Sci. Camb., 94, 69-88.

Farin, C.; Moeller, C.; Mayan, H.; Gamboni, F.; Sawyer, H. and Niswender, G. (1988): Effect of luteinizing hormone and human chorionic gonadotrophin on cell population in ovine corpus luteum, Biol. Reprod., $38,413-421$.

Fukui, Y.; Itagaki, R.; Ishida, N. and Okada, M. (2001): Effect of different hCG treatments on fertility of estrusinduced and artificially inseminated ewes during the non-breeding season, J. Reprod. Dev., 47, 189-195.

Gómez-Bruneta, A.; Santiago-Morenoa, J.; Montorob, V.; Gardec, J.; Ponsb, P.; González-Bulnesa, A. and López-Sebastián, A. (2007): Reproductive performance and progesterone secretion in estrus-induced Manchega ewes treated with hCG at the time of AI. Small Ruminant Research, 71(1-3), 117-122.

Gonzalez-Bulnes, A.; Santiago-Moreno, J.; Cocero, M.J.; Souza, C.J.H; Groome, N.P.; Garcia-Garcia, R.M.; Lopez-Sebastian, A. and Baird, D.T. (2002): Measurement of inhibin A and follicular status predict the response of ewes to superovulatory FSH treatment, Theriogenology, 57, 1263-1272.

Hafez, E.S.E. (1974): Reproduction in farm animals, Lea and Febiger, Philadelphia.

Husein, M.Q.; Mohammed, M.A. and Dia, S.A. (2007): The effects of short or long term FGA treatment with or without eCG on reproductive performance of ewes bred out-of-season. American Journal of Animal and Veterinary Sciences, 2(1), 23-28.

Ishida, N.; Okada, M.; Sebata, K.; Minato, M. and Fukui, Y. (1999): Effects of GnRH and hCG treatment for enhancing corpus luteum function to increase lambing rate of ewes artificially inseminated during the non-breeding season, J. Reprod. Dev., 45, 73-79.

Kassem, R.; Owen, J.B., Fadel, (1989): Breeding activity in milking Awassi ewes under semi-arid conditions. J. Anim. Prod., 49: 89-93.

Kaya, S.; Kaçar, C.; Kaya, D. and Aslan, S. (2013): The effectiveness of supplemental administration of progesterone with GnRH, hCG and PGF2 $\alpha$ on the fertility of Tuj sheep during the non-breeding season, Small Ruminant Research, 113, 365-370.

Keane, M.G. (1975): Effect of age and plane of nutrition during breeding on the reproductive performance of Suffolk-X ewe lambs, Ir. J. Agric. Res., 14, 91-98.

Kelidari H.R.; Souri, M.; Shabankareh H.K. and Hashemi, S.B. (2010): Repeated administration of hCG on follicular and luteal characteristics and serum progesterone concentrations in eCG-superovulated does, Small Ruminant Research, 90 (1-3), 95-100.

Kittot, R.J.; Stellflug, J.N. and Lowry, S.R. (1983): Enhanced progesterone and pregnancy rate after gonadotropin administration in lactating ewes, J. Anim. Sci., 56 (3), 652-655.

Knights, M.; Baptiste, Q.S.; Dixon, A.B.; Pate, J.I. and Marsh, D.J. (2003): Effects of a dosage of FSH vehicle and time of treatment on ovulation rate and pro-lificacy in ewes during the anestrous season, Small Rumin. Res., 50, 1-9. 
McNatty, K.P.; Gibb, M.; Dobson, C.; Ball, K.; Coster, J.; Heath, D. and Thurley, D.C. (1982): Preovulatory follicular development in sheep treated with PMSG and/or prostaglandin, J. Reprod. Fert., 65, 111-123.

Menchaca, A. and Rubianes, E. (2004): New treatments associated with timed artificial insemination in small ruminants. Reprod. Fertil. Develop., 16, 403-413.

Naohisa, I.; Midori, O.; Kazuhide, S.; Mayuko, M. and Yutaka, F. (1999): Effects of GnRH and hCG treatments for enhancing corpus luteum function to increase lambing rate of ewes artifi-cially inseminated during the non-breeding season, J. Reprod. Dev., 45, 73-90.

Nephew, K.P.; C'ardenas, H.; McClure, K.E.; Ott, T.L.; Bazer, F.W. and Pope, W.F. (1994): Effects of administration of human gonadotropin or progesterone before maternal recognition of pregnancy on blastocyst development and pregnancy in sheep, J. Anim. Sci., 72, 453-458.

Oliveira, C.A.; Dias, L.M.K.; Paes de Barros, M.B.; Viau, P.; Nicolau, S.S. and Sales, J.N.S. (2010): Effect of hCG on follicular dynamics in Santa Ines ewes submitted to ftai, Acta Scientiae Veterinariae, 38 (Supl 2), 675-821.

Santos, J.E.P.; Thatcher, W.W.; Pool, L. and Overton, M.W. (2001): Effect of human chorionic gonadotropin on luteal function and reproductive performance of high-producing lactating Holstein dairy cows, J. Anim. Sci. 79, 2881-2894.

Santos, I.W.; Binsfeld, L.C.; Weiss, R.R. and Kozicki, L.E. (2010): Fertility Rates of Ewes Treated with Medroxyprogesterone and Injected with Equine Chorionic Gonadotropin plus Human Chorionic Gonadotropin in Anoestrous Season, Veterinary Medicine International, 497-511.

Schmit, E.J.P.; Barros, C.M.; Fields, P.A.; Fields, M.J.; Diaz, T.; Kluge, J.M. and Thatcher, W.W. (1996): A cellular and endocrine characterization of the original and induced corpus luteum after administration of a gonadotropin-releasing hormone agonist or human chorionic gonadotropin on day five of the estrous cycle, J. Anim. Sci. 74, 1915-1929.

Swanson, L.V. and Young, A.J. (1990): Failure of gonadotropin-releasing hormone or human chorionic gonadotropin to enhance the fertility of repeat-breeder cows when administered at the time of insemination, Theriogenology, 34 (5), 955-963.

Wildeus, S. (2000): Current concepts in synchronization of estrus sheep and goats, J. Anim. Sci., 77, 1-14.

Wilmut, I.; Sales, D.J. and Ashworth, C.J. (1985): The influence of variation in embryo stage and maternal hormone profiles on embryo survival in farm animals, Theriogenology, 23, 107-119.

Zamiri, M.J. and Hosseini, M. (1998): Effects of human chorionic gonadotropin (hCG) and phenobarbital on the reproductive performance of fat-tailed Ghezel ewes, Small Ruminant Res., 30, 157-161. 